\title{
Uso de las técnicas de modelaje y refuerzo positivo para la regulación de la aproximación física indiscriminada en una niña de 5 años ubicada en un programa de acogimiento familiar: estudio de caso único
}

\section{Use of Modeling and Positive Reinforcement Techniques to Regulate Indiscriminate Physical Proximity in a 5 year-Old Girl in Foster Care: Case Study}

Ileana Morera Vargas

Universidad Nacional, Centro de Investigación y Docencia en Educación, Costa Rica ileana.morera.vargas@una.ac.cr

Recibido: 15/09/2019-Aceptado: 03/06/2020

\begin{abstract}
Resumen
El objetivo de este artículo es describir el estudio de caso único de una niña de 5 años, víctima de maltrato físico, negligencia y abuso sexual, ubicada en un programa de acogimiento familiar. Según criterios del DSM-5, muestra rasgos de Trastorno de relación social desinhibida, con ausencia de reticencia para aproximarse e interaccionar con personas adultas-conocidas y desconocidas-, en los diferentes espacios vitales, lo cual afecta sus relaciones interpersonales y se constituye en un factor de riesgo frente a nuevas situaciones de abuso sexual infantil. Se trabajó con técnicas de modelaje y reforzamiento positivo, desde un enfoque de Terapia de Juego Cognitivo-Conductual
\end{abstract}


(TJCC) y un enfoque de terapia ambiental, a fin de modificar patrones inadecuados de manifestaciones de afecto y acercamiento físico. Se pretendió promover la satisfacción de su necesidad de contacto físico y emocional a través del acercamiento discriminado y positivo que pudiera constituirse en un factor protector y que facilitara la construcción de relaciones interpersonales asertivas.

Palabras clave: Estudio de caso único, modelaje, refuerzo positivo, aproximación física indiscriminada, terapia de juego cognitivo-conductual, terapia ambiental.

\begin{abstract}
The objective of this article is to describe a unique case study involving a 5 year-old girl, who was the victim of physical abuse, neglect and sexual abuse, in a foster care program. According to DSM-5 criteria, she presented with signs of Disinhibited Social Relation Disorder, an absence of reticence to approach and interact with adults, both known and unknown to her, in different surroundings, affecting her interpersonal relationships and constituting a risk factor for new instances of sexual abuse. We worked with modeling modalities and positive reinforcement, focused on Cognitive Behavioral Game Therapy, as well as a focus on environmental therapy, with the goal of modifying inappropriate patterns of affection and close physical contact and approach. We attempted to satisfy her need for physical and emotional contact through limited and positive proximity, which could then constitute a protective tool for her, and could facilitate the building of assertive interpersonal relationships.
\end{abstract}

Keywords: Unique case study, modeling, positive reinforcement, indiscriminate physical approximation, cognitivebehavioral game therapy, environmental therapy.

\title{
INTRODUCCIÓN
}

Según el Centro de Prensa de la OMS, en un comunicado emitido en junio del año 2013, la violencia física o sexual es un problema de salud pública que afecta a más de un tercio de las mujeres a nivel mundial (OMS, 2013).

\section{(ㅅ) (1) (2)}


En Costa Rica, en el 2015 fueron notificados 12941 casos de violencia intrafamiliar (Ministerio de Salud, 2015), dato que, por las características propias de la violencia, es una cifra inferior a la real.

El impacto de la violencia intrafamiliar tiene repercusiones que van más allá del daño que sufren las víctimas directas. Los niños y niñas que crecen siendo testigos en entornos caracterizados por escenas violentas, dinámicas estresantes y negligencia social pueden desarrollar malestar psicológico.

Según Purvis, Cross, y Lyons Sunshine (2007), el abuso directo e indirecto y el descuido impiden que los circuitos cerebrales de niños y niñas se desarrollen completamente; se afectan, así, sus capacidades emocionales (expresión, interpretación y autorregulación), atencionales y sociales (razonamiento y procesamiento de estímulos).

Según dichos estudios, el afecto y los cuidados parentales en los primeros años de vida son cruciales para el óptimo desarrollo físico, emocional y social de las personas. Cuando se ven obstaculizadas por un ambiente carente de atención, caracterizado por el maltrato y el trauma, su trayectoria evolutiva será distinta.

Al respecto, Rygaard (2008) y Bilbao (2015) anotan que la organización del comportamiento inicia en la vida intrauterina y en las experiencias relacionales de los primeros años de vida; etapas que modelan la organización de las neuronas y defininen, así, la arquitectura cerebral.

Concuerdan en que la base de los comportamientos constructivos se adquiere en el contacto físico y emocional con las figuras primarias. Cuando los niños y niñas sufren el impacto de la violencia y se desarrollan en un ambiente carencial, se altera la organización cerebral.

El daño más grave que sufren los niños maltratados se manifiesta en su incapacidad para vincularse de manera sana y constructiva con su entorno social. (Rygaard, 2008, p.15)

Además, Gray (2012) menciona que los niños y niñas que han vivido en un hogar inseguro y negligente pueden experimentar lo que ella denomina "hambre de contacto", cuyo resultado puede incluir diversas manifestaciones, tales como la no regulación en el contacto físico e invasión del

\section{(ㅇ)(1)(2)}


espacio personal de los otros individuos, por ejemplo, sentarse en los regazos y besar a una persona extraña.

Al respecto, el Manual Diagnóstico y Estadístico de los Trastornos Mentales DSM-5 (2014) presenta el trastorno de relación social desinhibido como un trastorno relacionado con experiencias traumáticas y situaciones reiteradas de estrés, caracterizado por la falta de reticencia en el acercamiento físico.

De lo anterior, puede concluirse que, al ser el contacto físico una necesidad imperante para el adecuado desarrollo infantil, éste debe constituirse en un factor crítico de atención en quienes son víctimas de violencia intrafamiliar o en aquellas personas que, por su condición e historia, no han aprendido una forma segura de satisfacer la necesidad humana de afecto y de contacto.

En el presente caso clínico, la niña presentaba falta de reticencia en el acercamiento con personas conocidas y desconocidas a fin de satisfacer su necesidad de contacto, lo cual se constituyó en un factor de riesgo frente a nuevas experiencias de abuso sexual infantil.

Se presenta el uso del modelaje y refuerzo positivo, desde la terapia de juego cognitivo-conductual y la terapia ambiental, como alternativa terapéutica para modelar formas seguras de acercamiento físico, a fin de favorecer una de las necesidades básicas del ser humano: el contacto.

El primer apartado pretende contextualizar el caso clínico, resume datos relevantes de la paciente y el motivo de referencia por parte de la ONG; describe la formulación del caso clínico, historia clínica, situación problemática de la paciente, evaluación clínica y diagnóstico. Después se describe la intervención realizada en sus dos momentos claves: a) descripción de las premisas teóricas, y b) la intervención psicoterapéutica realizada. Al final, se resumen los resultados obtenidos y la discusión del caso, para culminar con una propuesta científica en el cuarto apartado.

\section{(c)(1)(2)}




\section{CONTEXTUALIZACIÓN DEL CASO CLÍNICO}

\section{Datos relevantes de la paciente y motivo de referencia}

La paciente es una niña de 5 años ubicada en una alternativa de acogimiento familiar de una ONG desde hace 2 años. Ha estado en 3 familias distintas desde su ingreso, ha residido con la familia actual durante el último año.

Previo a la institucionalización, residía con su madre y su padrastro, de quienes fue separada por la denuncia de la niña de agresión física severa y negligencia en el cuido sexual.

En el momento de la evaluación se encontraba en un proceso legal de declaratoria de abandono, por lo que su situación familiar futura no estaba definida.

La intervención se realizó a solicitud de la ONG, encargada de la alternativa de acogimiento familiar, al considerar el caso como prioritario, debido a la ausencia de regulación en la aproximación física y la vulnerabilidad que esto implicaba.

\section{Formulación del caso clínico}

La niña presentaba dificultades en su forma de relacionarse con otras personas, debido a necesidades afectivas (contacto físico y emocional) y patrones cognitivos inadecuados sobre las manifestaciones de afecto y acercamiento físico con seres conocidos y desconocidos, lo que ha incidido en la adquisición de patrones conductuales inadecuados para dar y recibir afecto.

Según referencia de la familia (con quien reside en el momento del estudio) y de la ONG, las dificultades observadas se presentaron desde el inicio del acogimiento en los diferentes espacios vitales en que la niña interactúa (centro educativo, amistades, familia de acogimiento); conducta presente durante la intervención psicoterapéutica.

\section{(ㅇ)(1) (2)(2)}


Además, se observaron estrategias de afrontamiento como la evasión, el distanciamiento afectivo (desconexión situación-emoción) y la fantasía; parece teatral su conducta y evita inicialmente hablar de la situación conflictiva.

Los resultados de la evaluación clínica muestran que la niña presentaba, según el Manual Diagnóstico y Estadístico de los Trastornos Mentales DSM-5 (2014), rasgos de trastorno de relación social desinhibida.

Entre ellos, haber experimentado un patrón extremo de cuidado insuficiente (negligencia y cambios repetidos de sujetos cuidadores) y la aproximación física con individuos adultos extraños, caracterizada por la ausencia de reticencia para aproximarse e interaccionar, la disposición para irse con una persona adulta extraña con poca o ninguna vacilación, sin que dichos comportamientos se limitaran a la impulsividad.

Por esta razón, resultó urgente facilitar otras opciones para relacionarse que disminuyeran el impacto de las condiciones de abuso y negligencia a las que fue expuesta.

Al no tener un hogar estable y permanente, la conducta desinhibida, la erotización temprana y el manejo inadecuado de los límites fueron considerados factores de riesgo.

Dentro de los factores protectores se consideraron: el adecuado nivel intelectual de la niña, la relación positiva con la familia de acogimiento actual, la asistencia a un centro educativo y el apoyo de la ONG. Estos pueden favorecer el aprendizaje de nuevas formas de vincularse desde la protección y el respeto a las necesidades propias de su edad.

\section{Historia y análisis de la situación problemática}

La paciente procede de una familia con una dinámica caracterizada por la desintegración, violencia e inestabilidad afectiva (múltiples figuras de cuido). Hay denuncias de desnutrición, negligencia y agresión física por parte de una cuidadora, y violencia física grave y sexual de las figuras parentales.

Por dicha razón, se recomendó su ubicación en una alternativa de protección, tras considerarse que se encontraba en condición de vulnerabilidad.

\section{(c)(1)(2)}


Durante su estancia en la ONG, la niña ha estado en tres familias de acogimiento. En las dos primeras fue retirada por conflictos con la autoridad y comportamientos asociados a una demanda de atención excesiva.

La familia con quien reside en el momento de la evaluación reportó aproximación física indiscriminada con conocidos y desconocidos y comportamientos afectivos erotizados.

Desde el ingreso a la ONG se mantuvieron visitas quincenales con la progenitora, después de éstas se incrementó la búsqueda de atención con comportamientos sexuados y de aproximación física desregulada.

La ambivalencia y la incertidumbre han sido las características principales en la historia de la paciente en el momento de la intervención, tanto a nivel legal, como familiar y psicológico.

\section{Evaluación clínica y diagnóstico}

La evaluación psicológica clínica tuvo por objetivo clarificar los comportamientos referidos y el origen de la ausencia de reticencia en el contacto físico. Se indagó la edad maduracional, funcionamiento intelectual, situación emocional, conflictos internos y comportamientos presentados en los entornos familiar y educativo.

Estuvo basada en diversas fuentes y métodos: entrevista clínica, examen mental, observación clínica, revisión de fuentes colaterales y la aplicación de pruebas psicológicas. A continuación, se describen las pruebas aplicadas y sus resultados.

Para la medición de la edad maduracional se empleó el Test de la figura humana de Vanne Kindergarten, comúnmente usado para la valoración cognitiva y visomotriz en población entre los 3,4 y 10,9 años. En éste se muestra una edad maduracional (EM) de 5 años y 10 meses; 4 meses superior a su edad cronológica.

El Test de inteligencia no verbal TONI-2 permite una estimación del funcionamiento intelectual mediante la evaluación de la capacidad para resolver problemas abstractos de tipo gráfico (donde no media el uso del lenguaje),

\section{(ㅇ)(1)(2)}


ofrece un puntaje global. Se observó una capacidad adecuada para resolver problemas no verbales ( $\mathrm{Cl} 96)$, lo cual sugiere que su aptitud y potencial de aprendizaje son adecuados para la edad. Esto puede apreciarse, de manera congruente, en el desempeño durante todas las sesiones (logra reconocer figuras geométricas y asociarlas, reconoce colores y escribe su nombre).

Se aplicaron las pruebas proyectivas para personas menores de edad: Test de apercepción infantil (TAT-H) y Test de fábulas de Düss (empleados típicamente para conocer las emociones y conflictos internos), y juego diagnóstico. Se observó un funcionamiento verbal deficiente en la expresión de sentimientos, y en análisis, comprensión y descripción del entorno. Su juicio es concreto y limitado.

Durante su aplicación, se destacó el mecanismo de afrontamiento y de evitación de manera persistente ante imágenes que implicaran una figura masculina dominante, lo cual desencadenó angustia evidente. La construcción de escenas de una niña sola que busca que la cuiden es recurrente, mas no habla de eventos personales.

En el juego diagnóstico la niña dramatizó roles caracterizados por violencia intrafamiliar, escenas sexuales entre personas adultas, búsqueda reiterada de afecto físico y su satisfacción de manera inapropiada (erotizada, invasiva), temores (soledad, desprotección). En ocasiones, verbalizó que algunas de estas escenas fueron vivenciadas con la pareja progenitora.

Finalmente, se aplicó a los sujetos encargados de la familia de acogimiento y a la maestra, el Cuestionario de comportamiento infantil para edades de 4 a 16 años (Achembach T.M.), para conocer, de forma cualitativa, el comportamiento de la niña en los entornos familiar y escolar; se confirmó, así, la aproximación física indiscriminada y contacto físico inapropiado.

Los resultados mostraron que la información recabada en las fuentes diversas tienen congruencia de los datos entre sí, y permiten un grado adecuado de validez y confiabilidad.

\section{(c)(1)(2)}




\section{INTERVENCIÓN TERAPÉUTICA}

\section{Premisas teóricas}

En el estudio se emplearon el modelaje y el reforzamiento positivo en dos fases, a saber, a través de la terapia de juego cognitivo-conductual en la primera fase y mediante la terapia ambiental en la segunda fase. Las premisas teóricas de ambas se resumen a continuación.

\section{Terapia de juego cognitivo-conductual}

En relación con el aprendizaje infantil, Bilbao (2015) afirma:

El cerebro del niño está diseñado para aprender a través del juego. Cuando jugamos con un niño, este entra en modo aprendizaje; todos sus sentidos se centran en la actividad, es capaz de permanecer concentrado, de fijarse en tus gestos y en tus palabras y de recordarlas mucho mejor que cuando lo instruimos o le ordenamos. (p. 38)

En este sentido, en la psicoterapia con niñas y niños pequeños, el uso del juego resulta ser una herramienta idónea, pues ésta es la forma más significativa en la que en esta etapa de vida se aprende.

La terapia de juego cognitivo-conductual (TJCC) se basa en las raíces teóricas conductuales y cognitivas del desarrollo emocional y la psicopatología. Su adaptación a la intervención en niñas y niños preescolares ha recibido especial atención desde la década de los 80 y fue desarrollada para usarse en edades comprendidas entre los dos años y medio y los seis años; incorpora las terapias cognitivas, las conductuales y la de juego.

En este sentido] la terapia cognitiva puede ser modificada para emplearse con niños pequeños si se presenta de una forma que les resulte accesible; por ejemplo, mediante títeres, animales de peluche, libros y otros juguetes para modelar estrategias cognitivas. Con la aproximación del modelo (por ejemplo, el títere) puede expresar de manera verbal habilidades de solución de problemas o soluciones a problemas análogos a los enfrentados por el niño. (Schaefer, 2012, p. 316)

\section{(ㅇ)(1)(2) (2)}


La TJCC comparte algunos principios de la terapia cognitivo-conductual para personas adultas de forma adaptada, por ejemplo, el tiempo limitado (es breve), es estructurada, directiva, orientada al problema u objetivo y es psicoeducativa.

Se implementa, por lo general, en un escenario de cuarto de juego. Resulta diferente a las formas tradicionales de terapia de juego, mas comparte la dependencia de una relación terapéutica positiva, el juego como medio comunicativo y el mensaje de que el contexto terapéutico es un lugar seguro.

Se diferencia en el énfasis en metas, la visualización del juego como una forma de educación, el énfasis en la relación pensamiento-conducta y el rol activo del terapeuta.

La TJCC tiene 4 fases:

1. El establecimiento de metas.

2. La selección de los métodos, donde se incluye alguna forma de modelamiento como componente crucial. "En la terapia de juego cognitivo-conductual se utiliza el modelamiento para mostrarle al niño habilidades de afrontamiento adaptadas" (Schaefer, 2012, p. 218).

3. Las intervenciones (reforzamiento positivo y modelaje en este estudio particular).

4. La evaluación de resultados.

\section{Terapia ambiental}

Según Purvis, et al. (2007), los niños y niñas en situación de riesgo y con dificultades en la vinculación no están preparados neurológicamente para mantener conversaciones prolongadas, por lo que resulta eficaz el uso de los sentidos para activar el apego y nuevas formas de relacionarse.

En este sentido, además de las actividades lúdicas, como el juego y el dibujo, en un contexto terapéutico individual, recomiendan la participación de quienes ejercen la función parental (acompañamiento emocional y psicoeducativo), a fin de incorporar en casa métodos complementarios

\section{(ㅅ) (1) (2)}


que les estimulen de forma cotidiana y se beneficien de la práctica y refuerzo diario.

Rygaard (2008) refiere que la terapia ambiental parte de varias premisas. La primera consiste en que "el paciente tiene pocos o ningún límite personal para separarse del entorno. Por consiguiente, la personalidad es todo el espacio o la habitación física entera en la que el niño está presente las 24 horas del día" (p.146).

La segunda explica que la terapia sólo influirá en el niño o niña mientras la persona que le estimula esté presente físicamente en esta habitación, pues su habilidad como paciente para retener emocionalmente la imagen es limitada.

Para Rygaard (2008) "en terapia ambiental, el énfasis se centra en dotar de una compensación para paliar la disfunción emocional y sus consecuencias" (p. 147).

Al respecto, la intervención con la familia constituye el principal recurso de cuidado y soporte, donde se construye la identidad del niño o la niña. Ésta tiene la labor terapéutica de reestructurar en el paciente la relación con su propio ser y con el entorno, a partir de su incorporación en un sistema donde se modelan alternativas vinculares y se satisfacen sus necesidades de afecto, pertenencia y protección.

En la edad preescolar, las exigencias sociales son más evidentes y empiezan a dejar al descubierto la diferencia en la madurez emocional de esta niñez; por lo que los objetivos específicos, según la terapia ambiental, en este grupo etario, consiste en equilibrar las exigencias sociales a la capacidad de los niños y niñas, es decir:

Hacer el mundo lo suficientemente pequeño para que le sea comprensible. Centrarse sobre las exigencias de comportamiento, pero no en las exigencias afectivas. Enseñar a los otros cómo comportarse con el niño con un trastorno de apego reactivo. Crear una estructura firme, amable y simple para el contacto social. (Rygaard, 2008, p. 185)

\section{(ㅇ)(1)(2) (2)}




\section{Intervención terapéutica}

El caso se abordó en dos fases: la primera desde el enfoque de la terapia de juego cognitivo-conductual y la segunda desde la terapia ambiental. Las técnicas básicas empleadas fueron el modelaje y el refuerzo positivo, al considerarse como la forma natural de aprendizaje en la etapa de desarrollo en que se encuentra la paciente.

En este sentido, Schunk (2012, p. 118) hace referencia a la teoría del aprendizaje social propuesta por Albert Bandura:

Al observar a los demás, las personas adquieren conocimiento, reglas, habilidades, estrategias, creencias y actitudes. Los individuos también aprenden la utilidad e idoneidad de las conductas y las consecuencias de las conductas modeladas a partir de la observación de modelos. (p. 118)

Los objetivos generales del proceso psicoterapéutico se enfocaron en la reestructuración cognitiva de las expresiones de afecto, haciendo énfasis en el contacto físico, a fin de promover formas asertivas de intercambio afectivo y la discriminación y reticencia en el contacto con personas conocidas y desconocidas.

La primera fase consistió en 7 sesiones de psicoterapia individual abordadas desde la terapia de juego cognitivo-conductual, con el empleo de técnicas básicas del modelaje y del reforzamiento positivo.

En la primera sesión se buscó, mediante el juego con peluches y muñecos sexuados, generar el rapport requerido para el proceso de intervención e identificar las cogniciones de la paciente en relación con las expresiones de afecto y contacto físico. La niña modeló sus propios esquemas cognitivos de acuerdo con las expresiones de afecto y contacto físico caracterizados por el acercamiento invasivo y erotizado.

En las dos sesiones siguientes, se empleó el modelaje y refuerzo positivo a través del juego con muñecos: ante la reproducción de las cogniciones de la niña se modelaron otras alternativas para conectar afectivamente (abrazos cortos mediante la técnica 1-2, besos en la mejilla, agradecer lo

\section{(ㅅ) (1) (2)}


que nos gusta que suceda). Ante su imitación se dio refuerzo social. Se enfatizó cognitivamente en "formas agradables de dar y recibir cariño".

Durante la segunda sesión la niña aceptó, con interés, las opciones modeladas y las repitió ante la invitación: "¿quieres intentarlo de esta otra forma?". En la tercera, inició el juego repitiendo sus patrones cognitivos antiguos, mas recordó las nuevas alternativas modeladas y las corrigió. Del hogar se reportó que en casa las había practicado espontáneamente, en particular, la técnica del abrazo 1-2.

La cuarta sesión se realizó con la encargada de acogimiento, a fin de 1) explorar el comportamiento de la niña en el espacio familiar en las últimas semanas, 2) iniciar un acercamiento con dicho núcleo para facilitar posteriormente la fase 2,3 ) comentar las necesidades emocionales y de contacto de los niños y las niñas víctimas de abuso y negligencia, y 4) promover la satisfacción de dichas necesidades estimulando el modelaje y refuerzo de las formas asertivas trabajadas en las sesiones individuales.

En las sesiones 5 y 6 , se reprodujo la dinámica de las sesiones 3 y 4 incluyendo las técnicas de la casa y familia de muñecos. Se modelaron alternativas asertivas de contacto y se reforzó la implementación de las conductas modeladas.

En la sesión 5, la niña reprodujo la dinámica de la familia de acogimiento empleando discriminación física y formas asertivas de expresión de afecto. Se observó satisfacción ante refuerzo positivo.

En la sesión 6, la niña implementó la técnica del abrazo 1-2 con la terapeuta, usó espontáneamente el agradecimiento y el cuidado ante la enfermedad como formas de expresión de afecto, y verbalizó sus emociones de manera auténtica (no dramática).

La séptima sesión se realizó nuevamente con la encargada de la familia de acogimiento. Se buscó conocer la percepción de las personas que están a cargo de la niña sobre sus estrategias de contacto afectivo y regulación del contacto físico, y definir los objetivos de la segunda fase del proceso psicoterapéutico. 
Se reportaron avances respecto a la discriminación física, cumplimiento de rutinas y límites, y participación positiva en el hogar. Se anotó que desde la última sesión cesaron conductas sexualizadas con una barbie. La encargada mencionó que la niña respondió positivamente al modelaje, reproduciendo lo observado y esperando el refuerzo social, el cual satisface una necesidad afectiva: la aceptación.

La fase 2 se basó en el enfoque de la terapia ambiental. Ésta consistió en 4 sesiones con los miembros de la familia de acogimiento. Se les convocó con el encuadre concreto de conocer las razones del contacto inadecuado de la paciente y aprender "juntos" cómo satisfacer sus necesidades emocionales y físicas de una forma positiva.

A la primera sesión llegó la encargada de acogimiento acotando las dificultades de horario de los otros miembros para asistir a las sesiones. Se reforzó la pertinencia de la intervención familiar en el proceso psicoterapéutico y el aporte de dicha intervención para el desarrollo de la niña y bienestar integral de la familia. A las siguientes sesiones asistieron 3 de 4 miembros.

Se realizó una labor psicoeducativa sobre las características de desarrollo de los niños y niñas con vivencias de abuso y negligencia. Se trabajaron las expectativas y temores en relación con el acogimiento, y su lógica de crianza con su progenie biológica (aciertos y desaciertos).

Se incorporó el modelaje y el refuerzo positivo como técnicas para modificar la conducta en el hogar de manera consistente con lo trabajado de forma individual, específicamente, los comportamientos de falta de reticencia en el acercamiento físico y acercamientos inadecuados. Se fueron incluyendo nuevas formas de acercamiento físico para la satisfacción de las necesidades afectivas de manera asertiva (pertenencia, aceptación, validación, reconocimiento).

\section{RESULTADOS}

Desde las primeras sesiones del proceso se observó en la paciente la aceptación y repetición de la nueva cognición y la conducta modelada consecuente: ante la invitación: “¿quieres intentarlo de otra forma?”, la niña sonreía y lo repetía. Inicialmente, de manera inmediata al modelado con los muñecos, posteriormente, después de haber emitido la conducta

\section{(ㅅ) (1) (2) (2)}


invasiva con los muñecos (corrigiendo), luego de manera espontánea con estos mismos y, finalmente, de manera espontánea con las personas (terapeuta y familia de acogimiento).

Sus juegos evidenciaron cambios conductuales en relación con las manifestaciones afectivas pasando de falta de reticencia y conductas sexualizadas a expresiones asertivas de afecto; por ejemplo, pasó de desnudar a los muñecos y ponerlos a darse besos en la boca o entrecruzar sus piernas; a manifestaciones verbales de afecto, besos en la mejilla, juegos entre ellos, preparar comida y alimentarlos o cuidar a alguno que estaba enfermo.

En las relaciones interpersonales mostró cambios cognitivos y conductuales más adaptativos. Por ejemplo, cuando se le cuestionaba: “¿cómo crees que se sienten las personas cuando les muestras el afecto de esa manera?", respondía: "las personas se sienten mejor cuando las beso en la mejilla", "las partes íntimas son privadas y no debo tocarlas"; y procedía a emplear un nuevo repertorio conductual: beso en la mejilla, abrazos cortos, respeto de las partes íntimas, manifestación del afecto a través del cuido, la alimentación y el juego.

El involucramiento del sistema familiar en la segunda fase del proceso, a través de la terapia ambiental, facilitó la implementación de las técnicas más allá del espacio psicoterapéutico individual y se logró consistencia en la aplicación de las técnicas en dicho espacio vital y una estimulación cotidiana.

Se refirió el uso adecuado de la técnica de los abrazos 1-2 y la modificación de la conducta sexuada e invasiva, distancia física con personas desconocidas y, al final del proceso, logró dormir sola y verbalizar sentimientos relacionados con el amor y el dolor.

Vale mencionar que los nuevos patrones cognitivo-conductuales implementados por la niña promovían patrones relacionales positivos de acercamiento y aceptación por parte de los sujetos adultos, facilitando la satisfacción de las necesidades vinculares de aceptación y contacto afectivo, aspectos fundamentales para un adecuado desarrollo socioafectivo.

Los alcances del proceso se observaron más allá de los objetivos planteados: se notó, en la familia de acogimiento, el cuestionamiento de su lógica de crianza y la sensibilización con otras formas de expresión de afecto

\section{시(1)(2)}


(abrazos), más allá de las empleadas en la dinámica cotidiana previa al acogimiento de la niña (cumplir los deberes).

Por lo anterior, se evidencia que durante el proceso la niña logró reestructurar los pensamientos e ideas referente a las expresiones de afecto y acercamiento físico con personas conocidas y desconocidas (cambio cognitivo) y, por ende, un cambio en las conductas en el espacio familiar. Resulta interesante mencionar que, por referencia de la encargada de la familia de acogimiento, se reportaron cambios al respecto en el entorno escolar, aspecto no profundizado en el presente estudio.

La reestructuración cognitiva y el cambio conductual logrado pueden contribuir a aminorar el riesgo de abuso sexual en la niña en cualquier espacio familiar en donde se ubique posterior a la resolución legal, pues ésta posee una mayor instrumentalización para identificar cuáles son formas adecuadas de acercamiento físico y cuáles no; además de ampliar el repertorio de posibilidades para satisfacer sus necesidades afectivas de contacto, aceptación y pertenencia.

Dentro de los alcances del proceso, más allá del logro de los objetivos, se muestra la eficacia de la técnica, la cual desde la segunda sesión evidencia tener resultados positivos. En este sentido, el factor de riesgo pasa a constituirse en un factor protector.

En la familia de acogimiento, la implementación de las técnicas facilitó la reflexión sobre una nueva alternativa para la adquisición de nuevas conductas en dicho espacio vital.

Por otra parte, el corto tiempo de implementación de la intervención familiar resultó ser una limitante en el presente estudio, pues no permitió dar seguimiento a dudas o conflictos que pudieran presentarse en el proceso e influir en un posible abandono de las nuevas cogniciones, y volver, así, a los antiguos patrones cognitivo-conductuales. Por lo anterior, se desconoce el impacto del presente proceso a largo plazo. 


\section{LA SÍNTESIS DEL PROCESO PSICOTERAPÉUTICO DESCRITO ES RESUMIDO EN LA FIGURA 1.}

Figura 1: Resumen del proceso psicoterapéutico

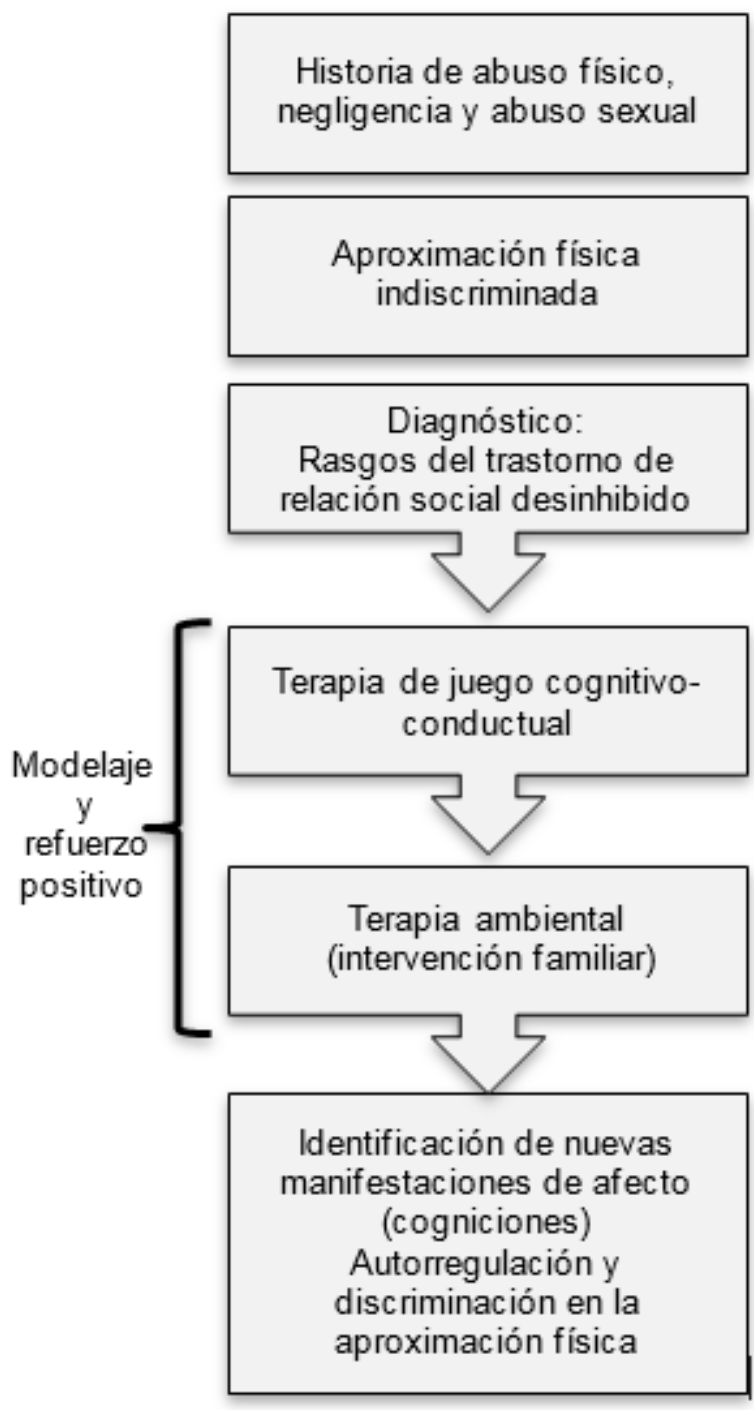




\section{DISCUSIÓN}

Según Bowlby (1995), el apego temprano es un comportamiento innato para aumentar la posibilidad de protección y sobrevivencia de niños y niñas. Se basa en la proximidad física y emocional entre este sujeto infantil y su figura primaria de protección.

Quienes han vivido violencia, negligencia y abuso a edades tempranas muestran incapacidad para vincularse de manera sana y constructiva (Rygaard, 2008). Por ello, en el interjuego entre el trauma, el apego y las necesidades de desarrollo, se necesita adaptar las acciones a lo que el niño y la niña necesitan, a fin de promover experiencias de vinculación más sanas y constructivas.

En la atención de esta población específica. Purvis et al. (2007) anotan la importancia de la participación, el juego y el elogio en el trabajo terapéutico. Al respecto refieren:

El juego transmite seguridad, por lo que resulta especialmente terapéutico para niños con necesidades especiales. Emplee el juego y los comentarios positivos siempre que pueda a lo largo del día.

Los niños también aprenden mejor cuando participan física y activamente: dialogando, tocando, mirando, moviéndose, hablando e interactuando. (p. 14)

En este sentido, el uso del modelaje y el refuerzo positivo dentro de la terapia de juego cognitivo-conductual y en la terapia ambiental (intervención familiar) resultaron altamente pertinentes con la condición y momento del desarrollo de la paciente para promover experiencias de vinculación positivas.

En el estudio de Prieto (2015), donde se realizó un análisis experimental de la eficacia de diferentes tipos de psicoterapia para la intervención de niños y niñas víctimas de maltrato infantil, se consideró que uno de los protocolos más eficaces en las mejoras a nivel de comportamiento estaba basada en la reestructuración cognitiva, al respecto plantea:

\section{(2) (1) (2) (2)}


El primer paso para ayudar a los menores y cuidadores es identificar las habilidades de afrontamiento cognitivas que emplean y la puesta en común de sus diálogos internos. Estos diálogos internos, sin embargo, pueden ser difíciles de capturar y compartir, sobre todo cuando están arraigados, son repetitivos e incluyen pensamientos estigmatizantes. Por ello, Cohen et al. (2006) recomiendan comenzar la reestructuración cognitiva con ejercicios no relacionados con el trauma. (p. 53)

En una etapa de desarrollo donde el lenguaje aún no es el vehículo principal de comunicación, el juego, según Purvis, et al. (2007) y Bilbao (2015), resulta ser el espacio más adecuado para el proceso de reestructuración cognitiva, lo que fue evidente en el tratamiento de la paciente en estudio, en quien se observó la aceptación de nuevos patrones dentro de un entorno seguro y un rapport adecuado.

Durante el proceso se logró incorporar, en el discurso y en la práctica de la paciente, otras formas reconocidas como expresiones afectivas, tales como el juego, el acompañamiento, la protección y el contacto físico regulado.

A nivel conductual se logró observar discriminación y autorregulación en el contacto físico con personas conocidas y desconocidas; ello permitió la satisfacción de las necesidades afectivas de contacto físico y emocional de una forma más segura y asertiva; promovió, así, el equilibrio cerebral y facilitó la atención de las necesidades de desarrollo propias de su edad.

Así mismo, la integración de los sujetos cuidadores en el proceso, donde se pretendió la toma de conciencia de sus propios modelos, la reflexión, reestructuración y la puesta en ejecución de nuevas acciones basadas en el nuevo conocimiento, resultó fundamental para el cambio cognitivo-conductual de la niña en estudio, al promover la implementación de los nuevos patrones en su cotidianidad.

Se concluye que, en el caso de niños y niñas víctimas de negligencia y abuso e historial de institucionalización y acogimiento familiar, resulta vital promover la discriminación física y la autorregulación, debido a la inestabilidad del entorno y a la vulnerabilidad de posteriores situaciones de abuso sexual. Ante esto, apelar a nuevos patrones cognitivos-conductuales, que

\section{(ㅇ)(1) (2)(2)}


fortalezcan formas asertivas de acercamiento físico y de intercambio afectivo, resulta no solo pertinente, sino prioritario.

\section{PROPUESTA CIENTÍFICA}

Según Rygaard (2009), la dinámica cerebral de los niños y niñas que han vivido situaciones de abuso y negligencia se encuentra orientada a la sobrevivencia, por lo que marginan su desarrollo físico, emocional y social. Sus necesidades básicas de protección y seguridad son violentadas; ello hace que vivan en una alerta constante que les limita avanzar.

El caso en estudio mostró que la niña trató de establecer contacto con las personas que le rodean en búsqueda de afecto, aceptación y protección, más carecía de un modelo cognitivo que le permitiera satisfacer su necesidad de una forma asertiva y segura. Si bien la evaluación realizada mostró que cuenta con una adecuada edad maduracional y capacidad cognitiva, sus habilidades emocionales y sociales estaban limitadas.

Sin embargo, las investigaciones, según Purvis et al. (2007), sugieren que es posible mejorar la bioquímica cerebral de los niños y niñas mediante sencillas intervenciones cognitivo-conductuales, con la finalidad de ayudarles a sentir seguridad, y ayudarles a conectar con sus padres, madres o sujetos cuidadores.

Por esta razón, el presente estudio pretendió abordar la falta de regulación en la aproximación física, a fin de facilitar la conexión asertiva y segura con las personas que le rodean y solventar sus necesidades emocionales. Para ello, se implementaron estrategias cognitivo-conductuales contextualizadas en: 1) su etapa de desarrollo (limitaciones en la comunicación verbal), 2) características propias a raíz de su historia de vida (negligencia, abuso sexual e institucionalización) y 3) su indiscriminación con su ambiente; es decir, su "personalidad es todo el espacio o la habitación física entera en la que el niño está presente las 24 horas del día" (Rygaard, 2008, p.146).

En este sentido, la implementación del modelaje y el refuerzo positivo en un contexto de intervención, donde se combinó la terapia de juego cognitivo-conductual y la terapia ambiental, resultó ser una alternativa acertada para la reestructuración cognitiva y conductual de la falta de regulación en

\section{(ㅇ) (1) (2) (2)}


el acercamiento físico, que inició en una dinámica simulada y segura (el juego) y trascendió a la cotidianidad de la niña (la dinámica familiar).

En el caso de niños y niñas víctimas de abuso y negligencia, que viven en condiciones de institucionalización o acogimiento, esto resulta fundamental. Más allá del aprendizaje logrado en el contexto psicoterapéutico individual, la posibilidad de ensayar en la vida cotidiana los nuevos patrones aprendidos para satisfacer sus necesidades de contacto físico y emocional de forma segura permite disminuir su vulnerabilidad y evitar que los rasgos de falta de reticencia en la regulación del contacto con sujetos conocidos y desconocidos pudiera convertirse en un trastorno. En este sentido, la propuesta de intervención del caso en estudio cobra gran pertinencia.

Sin embargo, la situación legal incierta y cambio recurrente de familia o persona cuidadora significa, para niños y niñas institucionalizados o en acogimiento, que en el corto o mediano plazo los modelos vinculares vuelven a cambiar, por tanto, es factible que, ante la ansiedad y proceso de cambio, retornen al modelo inicial.

Por esta razón, se propone ampliar la intervención implementada en el presente caso de estudio, a través de una labor psicoeducativa en los diferentes espacios vitales donde la niña interactúa cotidianamente, como lo son las familias de acogimiento, centro educativo y personal de instituciones vinculadas.

Esto facilitaría el mantenimiento y fortalecimiento de los nuevos modelos cognitivos y conductuales de vinculación, independientemente de los cambios de figuras de protección; es decir, para sostener los nuevos modelos aprendidos en el contexto individual (TJCC), hay que realizar una labor psicoeducativa integral, trabajando con los diferentes espacios vitales donde la niña interactúa, hasta fortalecerlos como sus patrones cognitivo-conductuales principales.

\section{REFERENCIAS}

Asociación Americana de Psiquiatría. (2014). Manual Diagnóstico y Estadístico de los Trastornos Mentales (DSM-5) (5 $5^{\mathrm{a}}$ ed.). Editorial Médica Panamericana.

\section{(ㅇ)(1) (2)(2)}


Bilbao, A. (2015). El cerebro del niño explicado a los padres ( $7^{a}$ ed.). Editorial Plataforma.

Bowlby, J. (1995). Una base segura, aplicaciones clínicas de una teoría del apego. Editorial Paidós.

Gray, D. D. (2012). Attaching in Adoption. Practical Tools for Today's Parents. Jessica Kingsley Publishers.

Ministerio de Salud. (2015). Casos notificados de violencia Intrafamiliar en boleta VE-01 (Dirección Vigilancia de la Salud, Costa Rica). https://www.ministeriodesalud.go.cr/index.php/vigilancia-de-la-salud/ analisis-de-situacion-de-salud/3035-notificacion-vif-costa-rica-2015/file

Organización Mundial de la Salud. (OMS, 2013, 20 junio). Informe de la OMS destaca que la violencia contra la mujer es un problema de salud global de proporciones epidémicas. Centro de Prensa. $\quad$ http://www.who.int/mediacentre/news/releases/2013/ violence_against_women_20130620/es/

Prieto, M. (2015). Eficacia de la terapia cognitivo conductual (TCC) y de la TCC-focalizada en el trauma en infancia maltratada [Tesis doctoral]. Universidad de Murcia, España. https://digitum.um.es/xmlui/ bitstream/10201/48124/1/TESIS\%20FINAL.\%20MACARENA \%20 PRIETO\%20LARROCHA.pdf

Purvis, K., Cross, D., y Lyons Sunshine, W. (2010). El niño adoptado. Ediciones Medici.

Rygaard, N. P. (2008). El niño abandonado. Guía para el tratamiento de los trastornos del apego. Editorial Gedisa.

Schaefer, C. E.. (2012). Fundamentos de terapia de juego ( $2^{\mathrm{a}}$ ed.). Manual Moderno.

Schunk, D. H. (2012). Teorías del aprendizaje, una perspectiva educativa ( $6^{\mathrm{a}}$ ed.). Pearson Educación.

\section{(ㅇ)(1) (2)}

